\title{
NOTES
}

\section{Polyurethane-Polystyrene Interpenetrating Polymer Networks Synthesized at Low Temperature: Effect of Temperature Change during Synthesis}

\author{
Doo Sung LEE* and Tae Sung PARK \\ Department of Textile Engineering, Sung Kyun Kwan University \\ Suwon, Kyungki 440-746, Korea
}

(Received September 4, 1990)

\begin{abstract}
KEY WORDS Polyurethane-Polystyrene IPN / Low Temperature Synthesis
/ Rate of Phase Separation / Rate of Network Formation / Competing

Processes / Miscibility /
\end{abstract}

It was illustrated that there are several factors in determining the degree of mixing in interpenetrating polymer network (IPN), such as the onset point of phase separation, the rate of phase separation, and the time of physical interlocking. These factors depend on the reaction conditions (such as initiator concentration, catalyst type and amount, crosslink density, synthesis temperature and synthesis pressure) and the thermodynamic value such as interaction parameter. ${ }^{1-6}$ Among these factors, the relative rate of the two competing kinetic processes of phase separation and network formation is the major factor of importance which is responsible for the morphology of the final product for given polymer pairs. When the rate of chemical reaction is very fast compared to that of phase separation, the interlocking of the two component polymers occurs in the early stage (i.e., at low conversion), the medium viscosity increases and the rate of phase separation is reduced. The resulting IPN's shows a highly homogeneous state. When the rate of chemical reaction is slow compared to that of phase separation, the opposite process is under control and IPN's with heterogeneous morphol- ogy are obtained where only partial interpenetration exists around the phase boundaries of the dispersed phase. Therfore, it is concluded that the domain size and the degree of partial interpenetration are dependent on the relative ratio of the rate of phase separation to that of network formation.

During past several years, we have tried to enhance the homogeneity of IPN by applying the reaction conditions such as synthesis pressure and temperature in an attempt to control the relative ratio of the rate of phase separation to that of chemical reaction. We were able to obtain a transparent, nearly molecular level mixture of the two components in the incompatible polyurethane-poly(methyl meth${\text { acrylate })^{2} \text { and polyurethane-polystyrene }}^{3-5}$ IPN's by applying high pressure (up to 20000 atm). The synthesis temperature, also, has direct and/or indirect effects on the onset point of phase separation, the rate of phase separation and the time of interlocking. When we synthesized the IPN at low temperature $\left(0^{\circ} \mathrm{C}\right)$, we were able to increase the homogeneity, because the medium viscosity increases at low temperature and thus the rate of phase separation decreases. ${ }^{6,7}$

* To whom all correspondence should be addressed. 
In this study, the reaction time was varied both at low temperature and at high temperature to study the effect of medium viscosity change during the synthesis on the rate of phase separation thus the homogeneity in resulting IPN. The relative ratio of the two competing kinetic processes of phase separation and network formation can be varied due to the change in medium viscosity before and after the gelation.

\section{EXPERIMENTAL}

The isocyanate-terminated polyurethane prepolymer was prepared by reacting 1 equivalent of poly(tetramethylene ether)glycol (PTMG, molecular weight 1,045) with 2 equivalents of $4,4^{\prime}$-Diphenylmethane diisocyanate (MDI) at $60^{\circ} \mathrm{C}$. Mixture of 1,4-butanediol (1,4-BD) and trimethylol propane (TMP) in $1: 4$ equivalent ratio was used as the crosslinking and chain-extending agent for the polyurethane (PU) network. Divinylbenzene (DVB, 55\% purity) was used as the crosslinking agent for the polystyrene (PS) network, its composition being $4 \%$ by weight in styrene monomer. The thoroughly mixed and degassed mixture of PU prepolymer, TMP/1,4-BD mixture (amount being adjusted to give total $\mathrm{NCO} / \mathrm{OH}$ ratio as 1 ), dibutyltin dilaurate as PU catalyst (T-12, $0.01 \mathrm{wt} \%$ in PU component mixture), styrene monomer, DVB, and benzoin as photo initiator $(0.3 \mathrm{wt} \%$ in PS component mixture) was charged between two glass plates sealed with a rubber O-ring and kept at $40^{\circ} \mathrm{C}$ for about $6 \mathrm{~h}$ for the polymerization of the PU component. With this condition, PU is believed to be polymerized almost but styrene monomer remains unreacted and is believed to be thoroughly dissolved in PU. Then the styrene monomer mixture was photo-polymerized by exposing to UV light in the temperature controlled chamber with the intention of observing the effect of temperature on the phase separation mainly during the photo-polymerization of PS component. The wavelength of UV light was $3,650 \AA$. The polymerization was started at $0^{\circ} \mathrm{C}$. After a certain reaction time, the reaction temperature was rapidly raised to $40^{\circ} \mathrm{C}$ during the photo-synthesis. The reaction time at $0^{\circ} \mathrm{C}$ was varied, but the total reaction time including the reaction times at $0^{\circ} \mathrm{C}$ and $40^{\circ} \mathrm{C}$ was maintained for $36 \mathrm{~h}$. The light intensity was different from the previous paper ${ }^{7}$ to reduce the reaction rate. The resulting theoretical molecular weight between crosslinks $\left(\bar{M}_{\mathrm{c}}\right)$ were 2000 for PS and 2,050 for PU, respectively. Each of the homopolymers having above $\bar{M}_{\mathrm{c}}$ was also prepared. The composition of the PU and PS was fixed at $50 / 50 \%$ by weight. After the reaction completed, samples were dried under vacuum at room temperature for 3 days before testing.

The dynamic mechanical properties were measured on a Du Pont 981-990 dynamic mechanical analyzer (DMA). The oscillation amplitude was $0.2 \mathrm{~mm}$ and the gap setting was $6.4 \mathrm{~mm}$. The scanning rate was $5^{\circ} \mathrm{C} / \mathrm{min}$ over a temperature range of -50 to $+130^{\circ} \mathrm{C}$.

Transmission electron micrographs were obtained on a JEM 100 CX (JEOL) electron microscope. The sample was stained with osmium tetraoxide $\left(\mathrm{O}_{5} \mathrm{O}_{4}\right)$.

\section{RESULTS AND DISCUSSION}

The $\tan \delta v s$. temperature plot of the IPN synthesized at $0^{\circ} \mathrm{C}$ and $40^{\circ} \mathrm{C}$ with varying reaction time is shown in Figure 1. The IPN synthesized at $40^{\circ} \mathrm{C}$ for $36 \mathrm{~h}$ shows inwardly shifted two distinct $T_{\mathrm{g}}$ 's of the PU- and PS- rich phases. But, the IPN synthesized at $0^{\circ} \mathrm{C}$ for $36 \mathrm{~h}$ shows broad transition and the low $T_{\mathrm{g}}$ shifts to a higher temperature and the high $T_{\mathrm{g}}$ shifts to a lower temperature, indicating that the intermixing between PU and PS in both phases is enhanced. As shown in the previous paper, ${ }^{6,7}$ the IPN prepared at low temperature shows the highly mixed state. It is because that the mobility of PS polymer chain is reduced due to high medium viscosity at low synthesis temperature, and the rate of phase separation 


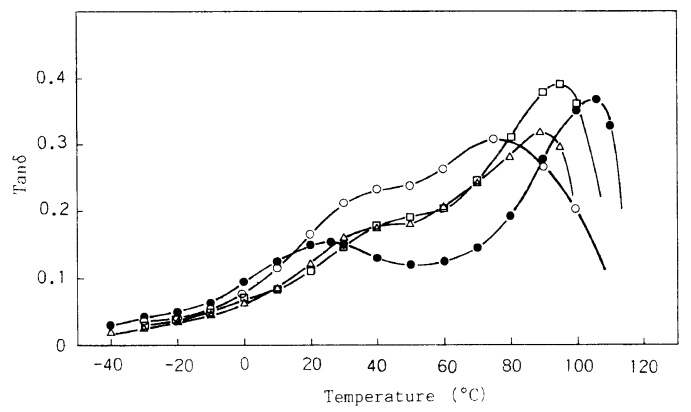

Figure 1. Dissipation factor $(\tan \delta) v s$. temperature of IPN's synthesized at varying reaction time at low temperature $\left(0^{\circ} \mathrm{C}\right)$ and high temperature $\left(40^{\circ} \mathrm{C}\right)$.

\begin{tabular}{ccc}
\hline & $0^{\circ} \mathrm{C}$ & $40^{\circ} \mathrm{C}$ \\
\hline$\bigcirc$ & $36 \mathrm{~h}$ & $0 \mathrm{~h}$ \\
$\square$ & 24 & 12 \\
$\triangle$ & 12 & 24 \\
$\bigcirc$ & 0 & 36 \\
\hline
\end{tabular}

becomes slow. On the other hand, the rate of network formation is slightly decreased at low synthesis temperature because of photopolymerization. Therefore, the reduced ratio of the rate of phase separation to the rate of network formation increases the homogeneity in the IPN synthesized at $0^{\circ} \mathrm{C}$ for $36 \mathrm{~h}$. Thus, we expect a gradual change in two $T_{\mathrm{g}}$ 's of the PU-and PS- rich phases by varying reaction time as each temperature and that is exactly what we observe in Figure 1 except for the IPN reacted for $12 \mathrm{~h}$ at $0^{\circ} \mathrm{C}$ and for $24 \mathrm{~h}$ at $40^{\circ} \mathrm{C}$. It is interesting that the IPN which was reacted for $12 \mathrm{~h}$ at $0^{\circ} \mathrm{C}$ and for $24 \mathrm{~h}$ at $40^{\circ} \mathrm{C}$ shows the better homogeneity than that reacted for $24 \mathrm{~h}$ at $0^{\circ} \mathrm{C}$ and $12 \mathrm{~h}$ at $40^{\circ} \mathrm{C}$. There seems to be another factor in this system which will be discussed later. The $T_{\mathrm{g}}$ 's of the PS-rich phase which were taken from the peak temperature in the $\tan \delta v s$. temperature plot are listed in Table I. The $T_{\mathrm{g}}$ s are rather high due to the instrument and the resting condition. The PU-rich phase $T_{\mathrm{g}}$ 's of the IPN's were difficult to measure due to broad transition.

To compare the degree of mixing with varying reaction condition, the $T_{\mathrm{g}}$ shift is
Table I. $T_{\mathrm{g}}$ and polymer composition (calculated from the Fox equation) of the PS-dominant phase for IPN with varying reaction time $(\mathrm{h})$ at $0^{\circ} \mathrm{C}$ and $40^{\circ} \mathrm{C}^{\mathrm{a}}$

\begin{tabular}{ccccc}
\hline \multirow{2}{*}{$\begin{array}{c}\text { Time at } \\
0^{\circ} \mathrm{C} / \mathrm{h}\end{array}$} & $\begin{array}{c}\text { Time at } \\
40^{\circ} \mathrm{C} / \mathrm{h}\end{array}$ & $\begin{array}{c}\text { High } T_{\mathbf{g}} \\
/ \mathrm{K}\end{array}$ & \multicolumn{2}{c}{ Composition/wt\% } \\
\cline { 4 - 5 } & & & PU & PS \\
\hline 36 & 0 & 348 & 0.36 & 0.64 \\
30 & 6 & 365 & 0.23 & 0.77 \\
24 & 12 & 368 & 0.21 & 0.79 \\
18 & 18 & 368 & 0.21 & 0.79 \\
12 & 24 & 362 & 0.25 & 0.75 \\
6 & 30 & 378 & 0.14 & 0.86 \\
0 & 36 & 379 & 0.13 & 0.87 \\
\hline
\end{tabular}

a $T_{\mathrm{g}}$ of the homopolymer: PU $284 \mathrm{~K}$, PS $399 \mathrm{~K}$.

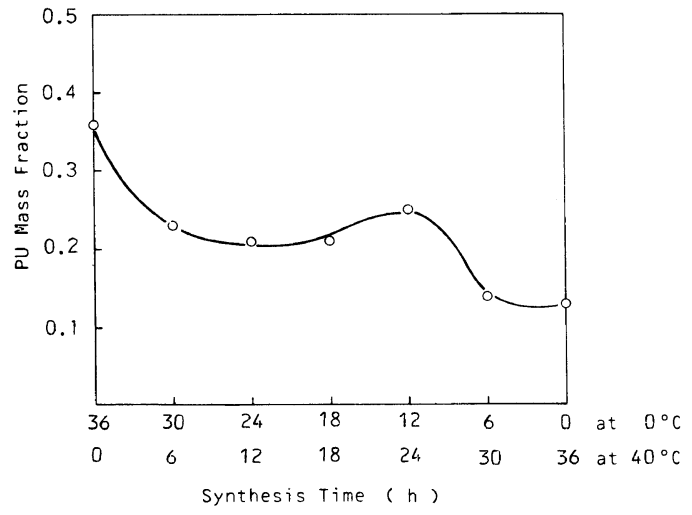

Figure 2. Calculated mass fraction of PU in the PSdominant phase of IPN's with varying reaction time at low temperature $\left(0^{\circ} \mathrm{C}\right)$ and high temperature $\left(40^{\circ} \mathrm{C}\right)$.

converted to the mass fraction of the PS and PU components within PS dominant phase by assuming that the Fox equation is valid in this system $^{1}$ :

$$
\frac{1}{T_{\mathrm{g}}}=\frac{w_{1}}{T_{\mathrm{g} 1}}+\frac{w_{2}}{T_{\mathrm{g} 2}}
$$

where $T_{\mathrm{g} 1}$ and $T_{\mathrm{g} 2}$ represent homopolymer $T_{\mathrm{g}}$ 's of the PU and PS, and $w_{1}$ and $w_{2}$ represent weight fractions. The resulting mass compositions in the PS dominant phase are shown in Table I and Figure 2.

Figure 2 shows the plot of reaction time variation of at $0^{\circ} \mathrm{C}$ and $40^{\circ} \mathrm{C}$ versus $\mathrm{PU}$ mass 
fraction in the PS dominant phase. The PU mass fraction increases from 0.13 to 0.36 with increasing the reaction time at $0^{\circ} \mathrm{C}$. The mass fraction 0.5 means the perfect mixing, so 0.36 means the $72 \%$ of degree of mixing of the component polymers. This means the fairly well mixed state. As was shown in Figure 1, when the reaction occurs at $0^{\circ} \mathrm{C}$ for $12 \mathrm{~h}$ followed by at $40^{\circ} \mathrm{C}$ for $24 \mathrm{~h}$, the mass fraction of PU in the PS dominant phase reaches a maximum value of 0.25 . This is not a estimated gradual change with increasing reaction time at $0^{\circ} \mathrm{C}$.

Above result can be illustrated as follows (see Table II). In the IPN synthesis, the polymerization starts from a single phase mixture of polyurethane and styrene monomer and the phase separation occurs as molecular weight increases. The relative ratio of the rate of network formation $\left(R_{\mathrm{p}}\right)$ to the rate of phase separation $\left(R_{\mathrm{s}}\right)$ is high at the initial stage of polymerization at low temperature. The high value of $R_{\mathrm{p}} / R_{\mathrm{s}}$ induces the more interlocking between two component polymers and thus it results in the highly mixed state of IPN. The value of $R_{\mathrm{p}} / R_{\mathrm{s}}$ can be varied as reaction undergoes and consequently conversion becomes high because of the increase in medium viscosity, the decrease in monomer and initiator concentration, the gelation and the vitrification at certain conversion. At high conversion, it is possible that the value of $R_{\mathrm{p}} / R_{\mathrm{s}}$ at low temperature can be reduced to be nearly equal to or smaller than the value at high temperature. As Gillham has illustrated from the relationship of time-temperature-transformation (TTT diagram) of the thermosetting system, ${ }^{8}$ the gelation retards or prevent the growth of a dispersed phase (the phase separation) and the vitrification retards chemical conversion (the network formation). After the gelation point, the temperature rise to $40^{\circ} \mathrm{C}$ induces the fast network formation and can avoid the possible vitrification at low temperature $\left(0^{\circ} \mathrm{C}\right)$ until the another vitrification occurs at $40^{\circ} \mathrm{C}$. For sample whose reaction times at
Table II. Magnitude change of the relative ratio of the rate of network formation and the rate of phase separation during the synthesis ${ }^{\mathrm{a}}$

\begin{tabular}{ccc}
\hline $\begin{array}{c}\text { Synthesis } \\
\text { temperature }\end{array}$ & $\begin{array}{c}\text { Ratio at } \\
\text { low conversion }\end{array}$ & $\begin{array}{c}\text { Ratio at } \\
\text { high conversion }\end{array}$ \\
\hline Low $T\left(0^{\circ} \mathrm{C}\right)$ & $R_{\mathrm{p}} / R_{\mathrm{s}}$ & $R_{\mathrm{p}} / R_{\mathrm{s}}$ \\
& $\vee$ & or $\wedge$ \\
High $T\left(40^{\circ} \mathrm{C}\right)$ & $R_{\mathrm{p}} / R_{\mathrm{s}}$ & $R_{\mathrm{p}} / R_{\mathrm{s}}$
\end{tabular}

$R_{\mathrm{s}}$, rate of phase separation.

$R_{\mathrm{p}}$, rate of network formation.

$0^{\circ} \mathrm{C}$ is $12 \mathrm{~h}$, it shows the maximum value and this result can be explained as follows: the gelation occurs before $12 \mathrm{~h}$ and the increase in temperature to $40^{\circ} \mathrm{C}$ renders the $R_{\mathrm{p}} / R_{\mathrm{s}}$ ratio to increase due to the devitrification for a while. On the other hand, $18 \mathrm{~h}$ at $0^{\circ} \mathrm{C}$ is enough for sample to reach certain conversion where is can vitrify even at $40^{\circ} \mathrm{C}$. In this case, there is no meaning to increase the temperature.

Although there is some experimental error range, this result shows clearly that the temperature variation could be very effective in reducing the possible microphase separation or secondary phase separation after the gelation to obtain the highly homogeneous IPN's. In the previous papers, ${ }^{6,7}$ we have focused only on the average value of $R_{\mathrm{p}} / R_{\mathrm{s}}$ variation during the whole period of synthesis and it has the higher value at low synthesis temperature.

The morphology via transmission electron microscopy also shows the effect of temperature change during synthesis on the degree of mixing and phase structure (Figure 3).

The IPN's prepared show the dual phase continuity and the domain sizes of PU in the range of hundreds to thousands of angstroms, which is the typical morphology of IPN system. ${ }^{1-3,6}$ Combining the microscopic observation with the dynamic mechanical behavior, it is shown that the domain size of PU decreases gradually with increasing degree of mixing.

Recently several papers ${ }^{2-7,9,10}$ have focused 


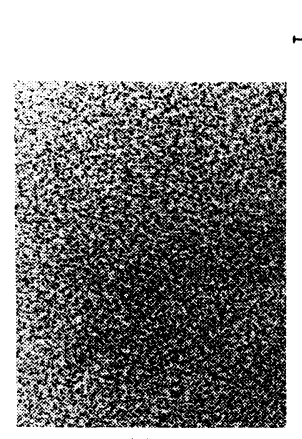

(a)

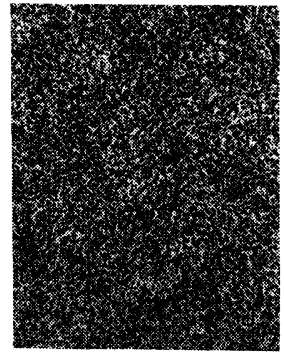

(c)

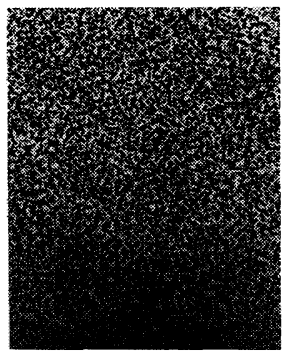

(b)

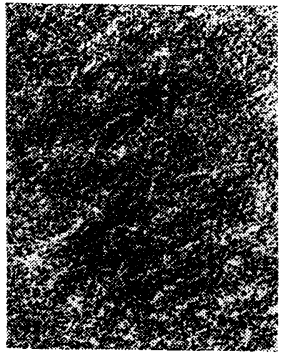

(d)
Figure 3. Transmission electron micrographs of the IPN's synthesized with varying reaction time (h) at $0^{\circ} \mathrm{C}$ and $40^{\circ} \mathrm{C}$. (a) 36,0 ; (b) 12,24 ; (c) 24,12 ; (d), 0, 36 .

on controlling the morphology and miscibility of polymer blends having either IPN structure or thermoset matrix modified with thermoplastics, by controlling the rate of phase separation and chemical reaction by means of physical condition variation during the synthesis such as temperature and pressure. From this study, it should be noted that a step change of reaction condition during synthesis is also helpful to get more homogeneous state. Undoubtedly, the optimal changing time of reaction condition depends on the rate of change of medium viscosity of component polymers during synthesis and the rate of two competing kinetic processes. In the further studies, we will investigate the possibility that the gradual change of reaction condition could be helpful to increase the homogeneity in polymer alloy including chemical reaction for preparation.

Acknowledgment. This reasearch was supported by the non-directed research fund of Korea Research Foundation, 1988.

\section{REFERENCES}

1. L. H. Sperling, "Interpenetrating Polymer Networks and Related Materials, "Plenum, New York, N. Y., 1981.

2. D. S. Lee and S. C. Kim, Macromolecules, 17, 268 (1984).

3. D. S. Lee and S. C. Kim, Macromolecules, 17, 2193 (1984).

4. D. S. Lee and S. C. Kim, Macromolecules, 17, 2222 (1984).

5. D. S. Lee and S. C. Kim, Macromolecules, 18, 2173 (1985).

6. B. S. Kim, D. S. Lee, and S. C. Kim, Macromolecules, 19, 2589 (1986).

7. D. S. Lee and T. S. Park, submitted for publication.

8. J. K. Gillham, Br. Polym. J., 17, 224 (1985).

9. K. Yamanaka and T. Inoue, Polymer, 30, 662 (1989).

10. K. Yamanaka, Y. Takagi, and T. Inoue, Polymer, 30, 1839 (1989). 